\title{
COVID-19-associated Ergonomical Impact and Modifications in the Current Anesthesia Practice
}

\author{
Joanna S Rodrigues ${ }^{1}$, Anil Parakh ${ }^{2}$
}

\begin{abstract}
"It is not the strongest of the species that survives, nor the most intelligent, but the one most responsive to change."-quoted Charles Darwin. While the world grapples with the coronavirus and adapts to it, it is imperative for us anesthesiologists to also modify our practice as per the demands of the situation. Despite the ergonomical impact, we must constantly strive to be one step ahead, to be wise enough to judge the feasibility and legitimacy of the working guidelines and accommodate the fleeting ever-changing nature of this disease.

Keywords: Anesthesiology, COVID-19, Ergonomics.

Research and Innovation in Anesthesia (2020): 10.5005/jp-journals-10049-0082
\end{abstract}

\section{INTRODUCTION}

The year 2020 not only heralds the beginning of a new decade but is also a harbinger of a new adaptation to living. Pandemics have afflicted civilizations throughout human history, with the earliest known outbreak occurring in $430 \mathrm{BC}$ during the Peloponnesian war. Many of these pandemics have had a significant impact on human society, from killing large percentages of the global population to causing humans to ponder larger questions about life. As time progressed, our knowledge of the diseases helped us in establishing health tools and policies that proved crucial in emerging victorious over such global catastrophes. The coronavirus pandemic currently continues to have an adamantine effect on mankind. Its claw-like effect has exposed our faulty and deficient healthcare system and one of the lesser unraveled aspects is its impact on the practice of anesthesia and its related ergonomics. Ergonomics is the study of people's efficiency in their working environment. "It is essentially fitting the workplace to the worker. The better the fit, the higher the level of safety and worker efficiency", quoted E Grandjean. The current kettle of fish has caused a disruption in our regular working pattern, crippled our proficiency and performance but has challenged us to rise to the situation.

If necessity is the mother of invention, then a crisis can surely be called a catalyst for change. The practice of anesthesia has been morally threatened and forced to be distorted right from the perioperative to the postoperative management of the patient.

\section{Preoperative}

\section{Preanesthetic Evaluation}

- The two main goals of preanesthetic evaluation are to allay the anxiety of the apprehensive patient scheduled for surgery and to deem the patient fit for the same. However, to minimize the contact period with the patient, this interaction is shortened. Thereby eliminating preoperative counseling and assuaging, limiting only to the patient assessment for surgery.

- Many of the patients seeking a preoperative assessment on an outpatient department basis are not evaluated for COVID-19. And hence the anesthesiologist conducting such examinations must be well protected. Many resort to the usage of a level-I PPE

\footnotetext{
1,2Department of Anaesthesiology, Global Hospital, Parel, Mumbai, Maharashtra, India

Corresponding Author: Joanna S Rodrigues, Department of Anaesthesiology, Global Hospital, Parel, Mumbai, Maharashtra, India, Phone: +91 7406287383, e-mail: joannarodrigues@yahoo.com

How to cite this article: Rodrigues JS, Parakh A. COVID-19-associated Ergonomical Impact and Modifications in the Current Anesthesia Practice. Res Inno in Anesth 2020;5(2):39-42.

Source of support: Nil

Conflict of interest: None
}

and the entire outfit (sometimes also a spacesuit look) might further add to the woes of the already fearful patient.

- Due to the fear of aerosol spread and scatter, airway examination particularly the Mallampati examination is omitted and the risk of an unanticipated difficult airway is increased.

- Masks worn by most patients are perfect masquerades failing to give us a rough configuration of the facial anatomy and dental alignment. Most patients themselves hesitate and fear lowering their masks and face shields to cooperate for such examinations. Airway examination that was mandatory and imperative to a meticulous anesthetic examination now becomes dispensable.

- Respiratory and cardiovascular auscultation is avoided and any underlying heart murmurs or rhonchi in an apparently healthy individual could be overlooked.

\section{Consent}

- Solitary visits to the hospital by patients are encouraged and hence obtaining consent from a patient (who is not compos mentis or who may not be able to comprehend and grasp the gravity of any given situation) becomes challenging.

- Some critically sick patients admitted in the intensive care unit (ICU) or on the ventilator may require emergency surgeries and in such patients, consent is obtained from the relatives telephonically or via a video call. Lapses in such communication and misconstrued information could have future medicolegal implications for the anesthesiologist. All video consents can 
be recorded and dispatched along with the patient files. Video proceedings may be required to have disclaimers stating that there could be elements of information that may not be communicated.

- In such times of affliction and grief, there have been instances whereby patients have sued the hospital for alleged hospitalacquired COVID-19 infection. Hence, many hospitals especially the anesthesiology teams have now resorted to a mandatory signing of a "special COVID-19" form. It is recommended that every hospital makes it mandatory to sign the Release of liability document which is like a blanket statement whereby the hospital including the surgical and anesthesiology staff will not be held liable for such litigations.

\section{Communication}

- "Between what is said and not meant, and what is meant and not said, most of love is lost."-quoted by Khalil Gibran.

- Communication is fundamental to good teamwork, increasing efficacy, and reducing errors in the workplace. Due to the current scenario, the communication barriers have increased such as noise dampening due to the personal protection equipment (PPE) kit, lack of a smartphone with video calling facilities (especially in developing countries like India), separation in time or location, lack of visual clues (body language), and interference. The richest communications with most information transfer are likely to be face-to-face interactions allowing for the influence of verbal and non-verbal communication which is absent in the COVID era.

\section{Investigations}

- Certain investigations have become mandatory during this pandemic.

- HRCT is considered highly superior to the COVID-19 antigen testing (due to its high false-negative and false-positive probabilities). It is also used as a screening and triage tool in many hospitals while the antigen test results are awaited. However, the high cost of such a test could be burdensome for the economically challenged. It has also been a bone of contention among the surgeons who question the cost factor and authenticity of such a test since it has been diagnostically less conclusive especially during the initial phase of the disease.

- RT-PCR COVID-19 test. The reliability of this test is highly questionable as there have been many instances of falsepositive and false-negative cases. The validity of this test has been set to 48 hours as per individual hospital protocols. However, sometimes due to insurance-related issues or due to other unforeseen reasons, the surgery may be scheduled 48 hours after the COVID test has been done which jeopardizes the whole prospect. Moreover, preoperatively a patient's report may be negative for COVID-19, but later he/she may become positive due to the incubation period of the disease or maybe hospital-acquired which may go undetected.

- Hence, it is best to consider and follow a working plan assuming the patient is COVID-19 positive despite the report. Based on such a negative report and to aid cost-cutting in our country, many institutions limit the use of PPE only to the surgeons, assisting nurses, and anesthesiologists, thereby putting the circulating staff, the housekeeping staff at risk.

- For those cancer surgeries involving head and neck which demand awake fiberoptic intubation, two negative COVID tests 5 days apart (with the patient in self-quarantine or hospital admission) along with HRCT correlation is a prerequisite.

- TrueNat antigen test is a rapid antigen test, now available, the results of which can be obtained within 45 minutes. This could be a benison, especially in all the emergency surgeries.

- Unfortunately, to prevent aerosolization, certain tests such as the pulmonary function tests which could be prudent and essential in a patient with respiratory issues are avoided. Treadmill tests done on a patient with a face mask could lead to erroneous results. It is deplorable that tests and tools that were designed to provide a guarded anesthesia plan are now omitted. In many circumstances, we have been forced to work, preparing, and expecting the worst-case scenario. Herein comes the quintessential task of maintaining the delicate balance and relaying the information about unrealistic optimism and unrealistic pessimism concerning the benefits and risks of the surgery with regards to anesthesia.

\section{Preoperative Optimization}

- As per most hospital protocols, relatives or bystanders of patients are not permitted inside hospital premises and patients often have to be alone entirely dependent on an already deficient nursing team. The presence of even one family member can be very assuring to the patient.

- Preoperative medications particularly sedatives such as benzodiazepines are avoided to prevent sedation which could be unsupervised and undetected leading to supplementation of high flow oxygen or an emergent situation. All these factors are responsible for a very anxious patient entering the operating room perhaps for the first time in their life.

- Preoperative nebulization is also avoided especially in positive pressure rooms to prevent aerosolization of droplets. This could hamstring optimization in patients with lung pathologies before general anesthesia.

- Truncating references to other specialty branches, to optimize the patient should be borne in mind to limit multiple doctorpatient exposures as well as to prevent unnecessary delay and rescheduling of surgery which could overshoot the validity time period of the COVID test and render it nugatory.

- Standard care of hygiene, social distancing, regular hand sanitization, and COVID etiquettes are expected to be practiced, while in hospital, both by doctors and patients.

- Intraoperatively, betadine-soaked throat packs and nasal packs are used to reduce the viral load. However, betadine gargles are now mandatory for all patients before any surgery.

\section{INTRAOPERATIVE}

\section{Preference of Anesthesia}

- Due to the increasing fear and paranoia, the trend nowadays favors day care surgeries. Most patients prefer immediate discharge to prevent contracting the virus. Keeping this in mind, peripheral nerve blocks are looked upon with disdain. General anesthesia is preferred for a quicker recovery and discharge 
although regional anesthesia would benefit the patient as well as the anesthesiologist.

- While administering general anesthesia, the overuse of neuromuscular blocking agents should be avoided to prevent residual effects in the postoperative period. Observation of patients in a postoperative anesthesia care unit (PACU) is bypassed and patients are fast-tracked and sent to the wards. Hence, it may be deemed necessary to resort to TOF monitoring to guide the use of neuromuscular blocking agents.

- There may be reassignment and shortages of resources such as blood products (due to shortage of volunteers visiting the hospitals and the imposed lockdown disrupting mobility and mode of travel) which may limit our supportive strategies.

- Contrary to the common belief, intraoperative use of dexmedetomidine can have prolonged respiratory effects along with profound hypotension and bradycardia crossing over in the recovery period and hence should be avoided. ${ }^{1}$

- Antitussive drugs, such as lidocaine, and dexmedetomidine, reduce the risk of coughing and minimize agitation on extubation and their use may be favored. ${ }^{2}$

- Polypharmacy is avoided as it may interact with many of the anti-retroviral drugs, antibiotics as well as targeted synthetic immunosuppressants and immune regulatory drugs that are used a part of the treatment plan for COVID patients.

- There is limited use of laryngeal mask airways in the current scenario as faulty seals or leaks are undesirable.

- TIVA vs inhalational: In this pandemic, TIVA is establishing its superiority in almost all aerosol-generating or triggering procedures. $^{3}$

- The major exceptional advantage of TIVA over inhalational anesthetic agents is minimal aerosol generation and can be provided using extension lines or infusion pumps which are at a safe distance from the patient.

- A generous antiemetic prophylactic regimen including at least two antiemetic agents along with dexamethasone, to lower the incidence of aerosolization of SARS-CoV-2 and to limit the need for subsequent staff interactions should be incorporated.

- To facilitate a fast-postoperative recovery with clear-headedness, the use of desflurane is encouraged.

\section{Ergonomical Impact}

- Donning a PPE has now become schtick. However, it comes with its own set of pitfalls. Some of them include:

- Fogging-suboptimal working conditions for the surgeon as well as the anesthesiologist.

- Lack of communication-the different layers of a PPE lead to reduced hearing, resulting in raised voice decibels which within the protective masks could be literally "breath-taking".

- Avoidance of air-conditioning in a positive pressure environment along with the multiple layers of PPE can lead to intense sweating, suffocation, and dehydration.

- Infrequent water and washroom breaks can be extremely uncomfortable.

Working under such conditions can lead to mental fatigue, irritability, and lead to lack of focus and coordination.

- Aerosol box - the aerosol box was designed to prevent splatter of the aerosols during intubation and extubation. However, it has been found to interfere with the ease of intubation and this could worsen in a difficult airway patient.

\section{Situation Awareness}

- "Sometimes the worst place you can be is in your own head"Christina Feist-Heilmeier. Given the ambiguous and obscure nature of this illness, fear has taken a stronghold in our minds.

- All our energies are focused on the prevention of virus contraction, that very often attention to other details and the nuances of anesthesia management takes a backseat.

\section{Duration}

- The turnover time for surgical cases has increased. Caution is to be exercised while permitting the entry of patient beds coming from the COVID ICU and wards into the sterile operating theater. This often leads to the transfer of patients on multiple beds and is time-consuming.

- Donning of PPE by all the OT personnel as well the incommodious working conditions have increased the duration of surgery and the ease of mobility of patients to and from the operating theaters.

- The turnover time between two surgeries has also increased by more than 45 minutes due to deep cleaning and fumigation of OT after each surgery.

\section{Fatigue, Burnout, and Emotional Well-being}

- The pandemic has amplified our already dismal doctor to patient ratio. Due to lack of medical health staff in general, COVID casualties and COVID affected healthcare workers, reassigned staff, the individual workload has increased. Long duty hours, extended duty schedules, and lack of vacation breaks have led to mental fatigue and burnout.

- Anesthesiologists above the age of 60 are exempted from working in COVID centers. However, obligated morally and due to an acute shortage of medical personnel, many of them render their services voluntarily. However, their clinical experience and expertise could be used for administrative purposes or they could be working behind the scenes with the younger forces taking lead.

- There is a constant nagging fright of contracting the infection and carrying the same home to their loved ones. This perpetual state of worry and the persistent violence and abuse against the medical community has affected our working temperaments.

- The outrageous pay cuts that have been imposed upon the medical fraternity despite the endangerments and risks involved are leading to an escalating resentment and indignation.

\section{Surgeries}

- To minimize the contact time with the suspected COVID-positive patient, many surgeons have clocked back and are favoring open surgeries. Though it may seem feasible and might be considered as the best option in the current scenario the patient benefit takes a hammering.

- Patients are reluctant to come for routine surgeries in hospitals that are treating COVID patients. Due to the social stigma attached to the disease as well as the deep-rooted fear, it will take some time to convince patients that hospitals are now safe for routine surgeries. 


\section{Postoperative}

\section{Pain Management}

- Epidural anesthesia and peripheral nerve blocks are avoided as patients seek an early discharge. This leaves us with very few tools in our armamentarium of pain management.

\section{Overall Expenditure}

- Due to the economical vagary, the cost of living has increased and so has the hospital expenses skyrocketed. Supplementary charges for PPE, gloves, N95 masks, and other COVID-related equipment have led to mammoth-sized bills for those availing operative care.

\section{ConcLusion}

As the renowned scientist Stephen Hawking quoted, "Intelligence is the ability to adapt to change". Our way of life has been disrupted and the world has been turned topsy-turvy by this invisible virus. All of us are grappling with this pandemic in numerous ways and struggling to survive. But there is a resounding consensus that henceforth, adaptability to this new way of living is imperative. We at the forefront as caregivers should constantly acclimatize to provide superlative healthcare services in any given predicamentthe patients' welfare being foremost.

\section{References}

1. Tung A, Fergusson NA, Ng N, et al. Medications to reduce emergence coughing after general anaesthesia with tracheal intubation: a systematic review and network meta-analysis. $\mathrm{Br} J$ Anaesth. 2020(4):S0007-0912(20)30012-X. DOI: 10.1016/j.bja.2019.12.041.

2. D'Silva DF, McCulloch TJ, Lim JS, et al. Extubation of patients with COVID-19. Br J Anaesth. 2020;125(1):192-195. DOI: 10.1016/j. bja.2020.03.016.

3. Chokshi T, Channabasappa S, Vergheese DC, et al. Re-emergence of TIVA in COVID times. Indian J Anaesth. 2020;64(14):S125-S131. DOI: 10.4103/ija.IJA_554_20. 